Sharif University of Technology
Scientia Iranica
Transactions A: Civil Engineering
SCIENTIA
IRAN I CA

Research Note

\title{
Effects of shear deformation on mechanical and thermo-mechanical nonlinear stability of FGM shallow spherical shells subjected to uniform external pressure
}

\author{
M.A. Shahmohamadi and M.Z. Kabir* \\ Department of Civil Engineering, Amirkabir University of Technology, Tehran, Iran.
}

Received 2 February 2015; received in revised form 5 December 2015; accepted 9 April 2016

\author{
KEYWORDS \\ Shallow spherical \\ shells; \\ Functionally graded \\ materials; \\ Nonlinear stability; \\ Transverse shear \\ deformation; \\ Mechanical load; \\ Thermal load; \\ Snap through.
}

\begin{abstract}
In the present paper, the asymmetrical nonlinear response of a clamped functionally-graded shallow spherical shell is subjected to uniform external pressure. It considers the effects of thermal stresses by both of the theories: Classical Laminate Theory (CLT) and First-order Shear Deformation Theory (FSDT). Material properties are graded in the thickness direction according to the power-law distribution in terms of the volume fraction of the constituents. Mechanical and thermo-mechanical properties are assumed to be temperature-independent and linear elastic. All of the governing equations are derived by aid of first-order transverse shear deformation theory considering geometrical nonlinearity. The nonlinear differential equation system is solved by Galerkin method. Buckling and post-buckling analyses have been done according to one-term deformation mode by the closed-form relation of load-deflection that shows the equilibrium path. Parametric studies are conducted to bring out the effects of shear deformation on the equilibrium path in different geometries and boundary conditions. Numerical results are presented in graphical arrangement, showing the geometrical nonlinear equilibrium paths. The effects of shear deformation on the equilibrium path are considered by comparing the results of FSDT and CLT, and they are verified by nonlinear finite-element method.

(C) 2017 Sharif University of Technology. All rights reserved.
\end{abstract}

\section{Introduction}

Shallow spherical shells make one of the most important structural elements group in many engineering structures. They can find their applications in many industrial structures such as power-plants, aerospace structures, building constructions, underground structures, and shipping. One of the most important studies of shallow spherical shells is their nonlinear response to uniform external pressure. Because of this importance, many researchers have noticed nonlinear buckling of

*. Corresponding author. Tel: +982164543000

E-mail addresses: amin13228@aut.ac.ir (M.A.

Shahmohamadi); mzkabir@aut.ac.ir (M.Z. Kabir) such structural types. Many different theories exist in the literature with special hypothesis. The classical shell theory is based on the thin shell theories, and it can be generalized to thin and thick shells by means of higher-order shear deformation theories. All of these theories can be compared to experimental results for validation. Yamada et al. [1] did an experimental investigation of the buckling of the clamped thin, shallow spherical shells under uniform external pressure in thermo-vacuumed process considering distributed geometrical imperfection. There are several theories for analysis of shells in the literature. Some of them are based on thin shell theories and the others can make accurate results for thin and moderately thick shells like first-order and higher-order shear 
deformation theories. According to this important difference, to increase the accuracy of results, especially in moderately thick shells, many studies on shells are based on these theories. Buckling of isotropic firstorder shear deformable shallow spherical shell by an analytical modified interaction method in boundary value problem was studied by $\mathrm{Li}$ et al. [2]. Lei et al. [3] presented an explicit fundamental solution of isotropic shallow spherical shells. Buckling modes of anisotropic cylindrical shells and sandwich spherical caps employing subspace interaction methods were studied by Cohen [4]. The equation of in-plane buckling and free vibration of anisotropic doubly curved shells on the basis of Flıgge's approximations incorporating shear deformation effects were investigated by Carrera [5]. Asymptotic buckling analysis of imperfect shallow spherical shells supported by nonlinear elastic foundation employing the asymptotic interaction method was investigated by Nie [6]. The requirements for progress, structural proficiency in many engineering industries such as nuclear power-plants or aerospace structures resulted in developing a new class of materials called composite materials. Using this kind of material makes it possible to obtain different aims (i.e., mechanical and thermo-mechanical resistance and stability) by means of different sub-materials for unit structure simultaneously. One of the most important of these kinds of material is laminated materials like plates and shells laminated by orthotropic layers. Because of the existence of weak surface between two layers, transverse shear deformation is so important in this kind of structures. So, a higher-order transverse shear deformation theory of laminated elastic shells was investigated by Reddy and Liu [7] by using a modified Sander's theory, which accounts for parabolic distribution of transverse shear strains through the thickness of the shells. Also, Ferreira et al. [8] investigated the Modelling crossply laminated elastic shells by a Higher-order theory. To progress structural proficiency of laminated composites, a new class of composite material has been developed, called Functionally Graded Materials (FGMs). FGMs are microscopically inhomogeneous, commonly manufactured from a blend of metals and ceramics with continuous composition gradations from pure ceramic on one surface with pure metal on the other surface. Many kinds of structural elements can be manufactured from FGMs like beams, spherical or cylindrical, or conical shells. Many researchers have studied these kinds of structures. Eslami and Hafezalkotob [9] studied linear thermo-mechanical buckling of simply supported FGM shallow spherical shells with temperature-dependent material. An analytical approach using the first-order shell theory of Love and Kirchhoff was employed. The calculus of variations is used for developing the geometrical linear equations in this study [9]. The non-linear axisymmetric re- sponse of FGM shallow spherical shells under uniform external pressure, including temperature effects using the classical shell theory and Galerkin method, was studied by Bich and Tung [10]. Also, the nonlinear dynamic response for FGM shallow spherical shells under low velocity impact on thermal environment was studied by Mao et al. [11]. FGMs can be used in different forms. Zenkour and Sobhy [12] studied thermal buckling of various types of FGM sandwich plates. These studies incorporate dynamic buckling of suddenly loaded imperfect hybrid FGM cylindrical shells. Buckling control of imperfect hybrid FGM plates with temperature-dependent material properties under thermo-electro-mechanical loads was studied by Shariyat [13,14]; FGMs are sometimes used with homogenous material simultaneously. Bending behaviour of FGM-coated and FGM-undercoated plates with two simply supported opposite edges and two free edges was studied by Chung and Chen [15]. Mechanical and thermal buckling analyses of functionally-graded plates were done by Zhao et al. [16]. In the present paper, nonlinear analysis of stability, consisting of buckling and post-buckling using first-order transverse shear deformation for FGM shallow spherical shells incorporating thermal effects has been done. It is possible to obtain the most accurate results for thin and thick spherical shells in comparison to Bich and Tung study [10] that is just for thin spherical shells by means of classical theory.

\section{Definition of geometry and material properties}

Consider a functionally-graded shallow spherical shell with radius of curvature $R$, based on radius $a$ and thickness $h$, as shown in Figure 1 . The shell is made from a mixture of ceramics and metals and is defined in spherical coordinate system $(\rho, \theta, \varphi)$ whose origin is located in the centre of the complete shell; $\varphi$ and $\theta$ are in the meridional and circumferential directions, respectively. The thickness of the shell is constant and the shell is shallow. So, it can be possible to make some changes in the coordinate system to make the following solution easier. So, $z$ is defined in the form

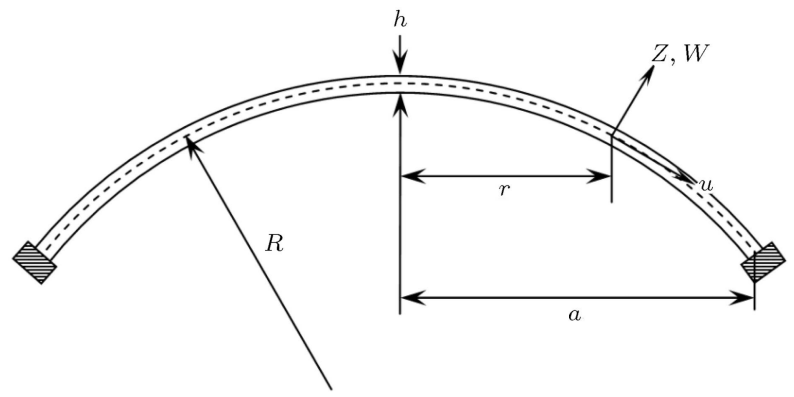

Figure 1. Functionally-graded shallow spherical shell. 
of $z=\rho-R$ and $z$ is perpendicular to the shell middle surface and represents the thickness direction and could change from $-h / 2$ to $+h / 2$.

According to the prior assumption, outer surface is made of pure ceramic; the inner surface is made of pure metallic materials; the material changes gradually along the thickness of the shell by the following simple power law distribution of ceramic and metal volume fractions as:

$$
V_{c}=\left(\frac{1}{2}+\frac{z}{h}\right)^{k}, \quad V_{m}=1-V_{c}
$$

where $V_{c}$ and $V_{m}$ are ceramic and metal volume fractions, respectively, which vary continuously through the thickness; $k$ is the volume fraction exponent that shows the kind of distribution of constituents depending on the form of manufacture of FGM; for instance, for $k=1$, the distribution of constituents is linear. Depending on the volume fraction of constituents, it is possible to define the material properties by linear rule of mixture as [10]:

$$
X_{\mathrm{eff}}=X_{c} V_{c}+X_{m} V_{m}
$$

$X_{\text {eff }}$ represents all of the effective mechanical and thermo-mechanical properties such as Young's modulus, $E$, density, $\rho$, thermal expansion, $\alpha$, and thermal conductivity, $K$. According to Eqs. (1) and (2):

$$
\begin{aligned}
{[E(z), K(z), \alpha(z)]=} & {\left[E_{c m}, K_{c m}, \alpha_{c m}\right]\left(\frac{1}{2}+\frac{z}{h}\right)^{k} } \\
& +\left[E_{m}, K_{m}, \alpha_{m}\right] .
\end{aligned}
$$

With reference to Eq. (3), the shell is assumed to be a mixture of ceramic and metal, where the mechanical properties depend on $z$, and it is measured along with the thickness direction and positive outward and varies between $-h / 2$ and $+h / 2$.

\section{The basic governing equations}

In this section, all of the basic equations, such as equilibrium, compatibility, and constitutive equations, will be derived. According to the FSDT, the normal and shear strains at a distance $z$ from the middle surface of the shell are [9]:

$$
\varepsilon_{r}=\varepsilon_{r}^{m}+z \chi_{r}, \quad \varepsilon_{\theta}=\varepsilon_{\theta}^{m}+z \chi_{\theta} .
$$

Due to the existence of axis-symmetrical loading, all of the in-plane shear forces, torsional moment, and derivatives of $\theta$ will be zero. So, normal strains of the middle surface of the shell (strain-displacement relations), considering the shallowness of the shell, are [10]:

$$
\begin{aligned}
& \varepsilon_{r}^{m}=u^{\prime}-\frac{w}{R}+\frac{1}{2}\left(w^{\prime}\right)^{2}, \\
& \varepsilon_{\theta}^{m}=\frac{u}{r}-\frac{w}{R}, \\
& \gamma_{r z}=\phi_{r}+w^{\prime}, \\
& \chi_{r}=\phi_{r}^{\prime} \quad \chi_{\theta}=\frac{\phi_{r}}{r} .
\end{aligned}
$$

$\chi_{r}$ and $\chi_{\theta}$ are middle surface bending curvatures and ()$^{\prime}$ is $d() / d r$. $\phi_{r}$ represents the rotation of the cross section of the shell where $u, v$, and $w$ are the meridional, circumferential, and radial displacements of the shell middle surface, respectively. Geometrical non-linearity in the case of small strain is accounted for. According to Hooke's law, the stress-strain relations for the spherical shell are:

$$
\begin{aligned}
\sigma_{r} & =\frac{E(z)}{1-v^{2}}\left(\varepsilon_{r}+v \varepsilon_{\theta}\right)-(1+v) \alpha \Delta T, \\
\sigma_{\theta} & =\frac{E(z)}{1-v^{2}}\left(\varepsilon_{\theta}+v \varepsilon_{r}\right)-(1+v) \alpha \Delta T, \\
\tau_{r z} & =\frac{E(z)}{2(1+v)} \gamma_{r z} .
\end{aligned}
$$

The forces and moment resultants based on the firstorder transverse shear deformation theory are:

$$
\begin{aligned}
& N_{r}=\int_{-h / 2}^{h / 2} \sigma_{r} d z, \\
& N_{\theta}=\int_{-h / 2}^{h / 2} \sigma_{\theta} d z, \\
& M_{r}=\int_{-h / 2}^{h / 2} \sigma_{r} \cdot z d z, \\
& M_{\theta}=\int_{-h / 2}^{h / 2} \sigma_{\theta} \cdot z d z \\
& Q_{r}=\beta \cdot \int_{-h / 2}^{h / 2} \tau_{r z} d z,
\end{aligned}
$$

where $\beta$ denotes the transverse shear correction coefficient that is equal to $5 / 6$ for isotropic shells [2], but it is different for functionally-graded materials and is given by [16]:

$$
\beta=\frac{5}{6-\left(v_{1} \cdot V_{1}+v_{2} \cdot V_{2}\right)},
$$

where $V_{1}$ and $V_{2}$ represent the volume fractions; and $v_{1}$ and $v_{2}$ are the Poisson ratios of the two constituents. 
Substituting Eqs. (3) and (6) into Eqs. (7), constitutive relations yield as:

$$
\begin{aligned}
& N_{r}=D_{1} \cdot\left(\varepsilon_{r}^{m}+v \cdot \varepsilon_{\theta}^{m}\right)+D_{2} \cdot\left(\chi_{r}+v \cdot \chi_{\theta}\right)-\frac{F_{m}}{1-v}, \\
& N_{\theta}=D_{1} \cdot\left(\varepsilon_{\theta}^{m}+v \cdot \varepsilon_{r}^{m}\right)+D_{2} \cdot\left(\chi_{\theta}+v \cdot \chi_{r}\right)-\frac{F_{m}}{1-v}, \\
& M_{r}=D_{2} \cdot\left(\varepsilon_{r}^{m}+v \cdot \varepsilon_{\theta}^{m}\right)+D_{3} \cdot\left(\chi_{r}+v \cdot \chi_{\theta}\right)-\frac{F_{b}}{1-v}, \\
& N_{\theta}=D_{2} \cdot\left(\varepsilon_{\theta}^{m}+v \cdot \varepsilon_{r}^{m}\right)+D_{3} \cdot\left(\chi_{\theta}+v \cdot \chi_{r}\right)-\frac{F_{b}}{1-v}, \\
& Q_{r}=\frac{\beta(1-v)}{2} D_{1}\left(\gamma_{r z}\right),
\end{aligned}
$$

where:

$$
\begin{aligned}
& D_{1}=\int_{-h / 2}^{h / 2} \frac{E(z)}{1-v^{2}} d z, \\
& F_{m}=\int_{-h / 2}^{h / 2} \Delta T \cdot E(z) \cdot \alpha(z) \cdot d z, \\
& D_{2}=\int_{-h / 2}^{h / 2} \frac{z \cdot E(z)}{1-v^{2}} d z, \\
& F_{b}=\int_{-h / 2}^{h / 2} \Delta T \cdot E(z) \cdot \alpha(z) \cdot d z, \\
& D_{3}=\int_{-h / 2}^{h / 2} \frac{z^{2} \cdot E(z)}{1-v^{2}} d z .
\end{aligned}
$$

Introducing Eq. (3) into Eqs. (10) yields the following:

$$
\begin{aligned}
D_{1}= & \frac{1}{1-v^{2}}\left(E_{m} h+\frac{E_{c m} h}{k+1}\right) \\
D_{2}= & \frac{E_{c m} h^{2}}{1-v^{2}}\left(\frac{1}{k+2}-\frac{1}{2(k+1)}\right) \\
D_{3}= & \frac{1}{1-v^{2}}\left(\frac{E_{m} h^{3}}{12}+E_{c m} h^{3}\left(\frac{1}{k+3}-\frac{1}{k+2}\right.\right. \\
& \left.\left.+\frac{1}{4(k+1)}\right)\right), \\
F_{m}= & \int_{-h / 2}^{h / 2} \Delta T \cdot\left(E_{m}+E_{c m} \cdot\left(0.5+\frac{z}{h}\right)^{k}\right) \\
& \cdot\left(\alpha_{m}+\alpha_{c m} \cdot\left(0.5+\frac{z}{h}\right)^{k}\right) \cdot d z,
\end{aligned}
$$

$$
\begin{gathered}
F_{b}=\int_{0 h / 2}^{h / 2} \Delta T \cdot\left(E_{m}+E_{c m} \cdot\left(0.5+\frac{z}{h}\right)^{k}\right) \\
\cdot\left(\alpha_{m}+\alpha_{c m} \cdot\left(0.5+\frac{z}{h}\right)^{k}\right) \cdot z . d z .
\end{gathered}
$$

The non-linear equilibrium equations of a perfect shallow spherical shell based on the first-order transverse shear deformation theory are given by $[2,10,11]$ :

$$
\begin{aligned}
& \text { (i) : } \frac{d\left(r N_{r}\right)}{d r}-N_{\theta}=0, \\
& \text { (ii) : } \frac{d\left(r M_{r}\right)}{d r}-M_{\theta}-r Q_{r}=0, \\
& \text { (iii) : } \frac{d^{2}\left(r M_{r}\right)}{d r^{2}}-\frac{d\left(M_{\theta}\right)}{d r}+\frac{r}{R}\left(N_{r}+N_{\theta}\right) \\
& \quad+\frac{d}{d r}\left(\frac{r d w}{d r}\right)+r q=0,
\end{aligned}
$$

where $q$ is uniform external pressure positive inwards. The first part of Eqs. (12) will be satisfied by introducing a potential force function $f$ as:

$$
N_{r}=\frac{f^{\prime}}{r}, \quad N_{\theta}=f^{\prime \prime} .
$$

From the constitutive relations Eqs. (9), one can write:

$$
\begin{aligned}
\varepsilon_{r}^{m} & =\frac{1}{E_{1}}\left(N_{r}-v \cdot N_{\theta}\right)-\frac{E_{2}}{E_{1}}\left(\chi_{r}\right)+\frac{F_{m}}{E_{1}}, \\
\varepsilon_{\theta}^{m} & =\frac{1}{E_{1}}\left(N_{\theta}-v \cdot N_{r}\right)-\frac{E_{2}}{E_{1}}\left(\chi_{\theta}\right)+\frac{F_{m}}{E_{1}} .
\end{aligned}
$$

The axsisymmetric geometrical compatibility equation of a shallow spherical shell is written as [10]:

$$
-\frac{1}{r} \frac{d\left(\varepsilon_{r}^{m}\right)}{d r}+\frac{1}{r^{2}} \frac{d}{d r}\left(\frac{r^{2} d\left(\varepsilon_{\theta}^{m}\right)}{d r}\right)=-\frac{\Gamma(w)}{R} \chi_{r} \chi_{\theta},
$$

where:

$$
\begin{aligned}
\Gamma() & =()^{\prime \prime}+\frac{1}{r}()^{\prime}=\frac{1}{r} \frac{d}{d r}\left(r \frac{d()}{d r}\right), \\
\Gamma^{2}() & =()^{(4)}+\frac{2}{r}()^{\prime \prime \prime}-\frac{1}{r^{2}}()^{\prime \prime}+\frac{1}{r^{3}}()^{\prime} \\
& =\frac{1}{r} \frac{d}{d r}\left(r \frac{d}{d r}\left(\frac{1}{r} \frac{d}{d r}\left(r \frac{d()}{d r}\right)\right)\right) .
\end{aligned}
$$

Substituting Eqs. (14) into Eq. (15), with the aid of Eqs. (5) and (13), yields the compatibility equation of a perfect FGM shallow spherical shell as:

$$
\frac{1}{D_{1}\left(1-v^{2}\right)} \Gamma^{2}(f)=-\frac{\Gamma(w)}{R}=\frac{1}{r} \phi_{r} . \phi_{r}^{\prime} .
$$


By inserting Eqs. (5), (9), and (17) into the second and third parts of Eqs. (12), the bending equilibrium equation is obtained as follows:

$$
\begin{aligned}
&-D_{i} \Gamma^{2}(\Phi)-D_{2}(1-v)\left(\frac{w^{\prime} w^{\prime \prime}}{r}-\frac{\phi_{r} \cdot \phi_{r}^{\prime}}{r}\right) \\
&-\frac{1}{R} \Gamma(f)-\frac{1}{r}\left(f^{\prime} w^{\prime}\right)^{\prime}=q \\
&-v\left(1-v^{2}\right) \frac{D_{2}}{D_{1}} \Omega\left(f^{\prime}\right)-v \cdot D_{2} \cdot w^{\prime} \cdot\left(\frac{r}{R}+\frac{w^{\prime}}{2}\right) \\
&+D_{i} \Omega\left(\phi_{r}\right)-\frac{\beta \cdot(1-v) \cdot D_{1}}{2}\left(r w^{\prime}+r \phi_{r}\right)=0,
\end{aligned}
$$

where:

$$
\begin{aligned}
& D_{i}=D_{3}-\frac{D_{2}}{D_{3}} D_{2}, \quad \Phi=\int \phi_{r} d r, \\
& \Omega()=r()^{\prime \prime}+()^{\prime}-\frac{1}{r}() .
\end{aligned}
$$

Eqs. (17) and (18) are the compatibility and equilibrium equations of functionally-graded shallow spherical shells which make three equations in terms of three dependent unknowns that are deflection of the shell $(w)$, rotation of the shell cross section $\left(\phi_{r}\right)$, and potential force function $(f)$. These three nonlinear differential equations are basic equations used to consider the nonlinear stability functionally-graded shallow spherical shells incorporating effects of thermal stresses and shear deformation by means of first-order shear deformation theory.

\section{General solution and boundary conditions}

In this section, an analytical approach is employed to consider the non-linear axis-symmetric stability of functionally-graded shallow spherical shells under uniform external pressure with and without thermal stresses and investigate the effects of transverse shear deformation. The Galerkin method [10] is adopted to solve the non-linear differential equation system given in Eqs. (18). By employing appropriate weighting functions which satisfy all of the static and dynamic boundary conditions, this method is actually an accurate method. Labelled Cases (1) and (2), as two investigated cases of boundary conditions, often are encountered in engineering applications $[2,10]$ :

- Case (1): The edge is clamped along the periphery and is freely movable in the meridional direction. This boundary condition is described by $[2,11]$ :

$$
\begin{array}{lll}
r=0 & w=W \quad w^{\prime}=0 & \varphi_{r}=0, \\
r=a & w=w^{\prime}=\varphi_{r}=0 & N_{r}=0 .
\end{array}
$$

- Case (2): The edge is clamped along the periphery and is freely immovable in the meridional direction. This boundary condition is described by $[2,11]$ :

$$
\begin{aligned}
& r=0, \quad w=W, \quad w^{\prime}=0, \quad \varphi=0, \\
& r=a, \quad w=w^{\prime}=\varphi_{r}=0, \quad N_{r}=N_{r 0},
\end{aligned}
$$

where $W$ is the maximum of deflection (i.e., out of plane deflection) located at the summit of the FGM shallow spherical shell and $N_{r 0}$ is a meridional reaction force of the edge which belongs to immovable edge. Considering the boundary conditions described by Eqs. (20) and (21), weighting functions for $w$ and $\phi_{r}$ are approximately assumed as follows [10]:

$$
w=W \frac{\left(a^{2}-r^{2}\right)^{2}}{a^{4}}, \quad \phi_{r}=C \frac{r\left(a^{2}-r^{2}\right)}{a^{4}},
$$

where $C / a$ represents the maximum rotation of the cross section of the shell. Solution form Eq. (22) is similar to the solution presented in [10] for thin FGM shells by aid of classical theory. Present solution is a generalization of describing a solution for thin and thick shells by aid of first-order transverse shear deformation theory.

Introducing Eqs. (22) into Eq. (17) and consecutive integration of the resulting equation yield a potential force function $f$ with:

$$
\begin{aligned}
f^{\prime}=- & \frac{\left(1-v^{2}\right) D_{1} W}{R \cdot a^{4}}\left(\frac{r^{5}}{6}-\frac{a^{2} r^{3}}{2}\right) \\
& -\frac{\left(1-v^{2}\right) D_{1} C^{2}}{a^{6}}\left(\frac{r^{7}}{96}-\frac{a^{2} r^{5}}{24}+\frac{a^{4} r^{3}}{16}\right) \\
& -\frac{C_{1} r}{2} \ln (r)+\frac{C_{2} r}{2}+\frac{C_{3}}{r},
\end{aligned}
$$

where $C_{1}, C_{2}$, and $C_{3}$ are constants of consecutive integration. Due to the existence of limited value of the stresses, strains, and forces at $r=0$, coefficients of $\ln (r)$ and $1 / r$, i.e. the constants $C_{1}$, and $C_{3}$, must be zero. The constant $C_{2}$ will be obtained by the aid of in-plane (i.e., Meridional) restrain boundary condition, i.e. $N_{r}(r=a)=N_{r 0}$. After determining the constants, potential force function, $f$, will be obtained, such that:

$$
\begin{aligned}
f^{\prime}= & -\frac{\left(1-v^{2}\right) D_{1} W}{R \cdot a^{4}}\left(\frac{r^{5}}{6}-\frac{a^{2} r^{3}}{2}\right) \\
& -\frac{\left(1-v^{2}\right) D_{1} C^{2}}{a^{6}}\left(\frac{r^{7}}{96}-\frac{a^{2} r^{5}}{24}+\frac{a^{4} r^{3}}{16}\right) \\
& -\frac{\left(1-v^{2}\right) D_{1} W}{3 . R} r+\frac{\left(1-v^{2}\right) D_{1} \cdot C^{2}}{32} r+N_{r 0} . r .
\end{aligned}
$$

For the movable boundary condition described in 
Eq. (20), $N_{r 0}$ must be zero. Substituting Eqs. (22) and (24) into Eqs. (18), while employing Galerkin method, yields a non-linear equilibrium path in the form of two relations as:

$$
\begin{aligned}
(1) q= & \frac{3 \cdot\left(1-v^{2}\right) \cdot D_{1} h C^{2}}{40 a^{4}}+\frac{\left(1-v^{2}\right) \cdot D_{1} h}{3 R^{2}} \\
& \left.+\frac{24 \cdot h \cdot N_{r 0}}{5 \cdot a^{2}}\right) \bar{W}-\left(\frac{32 h^{2}\left(1-v^{2}\right) \cdot D_{1}}{35 a^{2} R}\right. \\
& \left.+\frac{32 \cdot(1-v) \cdot h^{2} D_{2}}{5 a^{4}}\right) \bar{W}^{2}+\left(\frac{16 \cdot D_{i}}{a^{3}}\right) \cdot \bar{C} \\
& +\left(\frac{2 \cdot(1-v) \cdot D_{2}}{\left.5 a^{2}-\frac{3 \cdot\left(1-v^{2}\right) D_{1}}{112 \cdot R}\right) \cdot \bar{C}^{2}}\right. \\
& \left.-\frac{2}{R} N_{r 0}, \quad \begin{array}{c}
v \cdot D_{2} \\
21
\end{array}\right) \cdot \bar{C}^{2}-\left(\frac{16 \cdot v \cdot D_{2} h^{2}}{a^{2}}\right) \cdot \bar{W}^{2} \\
& \quad-\left(\frac{\beta \cdot a \cdot(1-v) \cdot D_{1}}{15}+\frac{8 D_{i}}{5 a}\right) \cdot \bar{C} \\
& +\left(\frac{4 \cdot \beta(1-v) \cdot D_{1} \cdot h}{15}\right) \cdot \bar{W}=0 \\
(2) &
\end{aligned}
$$

where $\bar{W}$ and $\bar{C}$ are dimensionless parameters of deflection described as:

$$
\bar{W}=W / h \quad \bar{C}=C / a .
$$

Using Eqs. (25), it is possible to determine the buckling loads and non-linear equilibrium paths of FGM shallow spherical shells under uniform external pressure with and without the thermal effects, considering the transverse shear deformation. The second part of Eqs. (25) is used to determine $\bar{C}$ in terms of $\bar{W}$. Substituting $\bar{C}$ obtained from the second part of Eqs. (25) into the first part yields an equilibrium path in form of $q-\bar{W}$ relation.

\section{Mechanical and thermo-mechanical Stability analysis}

In this section, nonlinear stability of functionallygraded shallow spherical shells, including buckling and post-buckling, by incorporating thermal stresses and transverse shear deformation, are investigated by exposition of equilibrium path. Figure 2 shows the schematic equilibrium path obtained from Eqs. (25). The equilibrium paths are stable, that is, the deflection is monotonically increasing when the pressure rises. The equilibrium path indicates that extremum points of $q(\bar{W})$ curve determine the unstable zone. Unstable

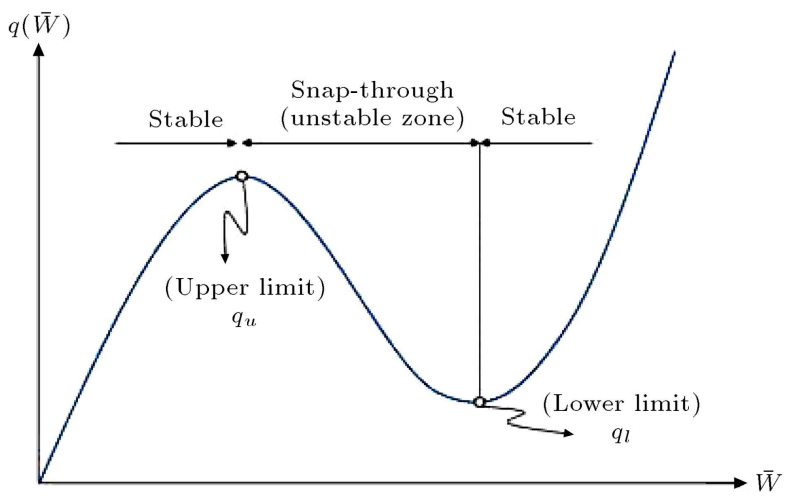

Figure 2. Schematic form of $q-\bar{W}$ relation.

zone is between the minimum and maximum points of $q(\bar{W})$ curve. In this zone, by increasing the inward deflection (i.e., inward deflection is positive), uniform external pressure is negative (i.e., pressure is outward). So, stiffness application is negative and snap-through behaviour will appear.

Considering constitutive relations in Eqs. (9) and compatibility equation (Eq. (15)), it is evident that the thermal stresses appear in terms of $N_{r 0}$ in first part of Eqs. (25). As mentioned, considering freely movable edge results, $N_{r 0}=0$, effects of thermal stresses appear when the boundary edge is immovable. The condition to express the immovability on the boundary edge, i.e. $u=0$ on $r=0$, is represented in an average form as:

$$
\int_{0}^{a} \frac{\partial u}{\partial r} d r=0
$$

From Eq. (5) and using Eqs. (13) and (14), the following relation can be obtained:

$$
\begin{aligned}
N_{r 0}= & \left(\frac{64(1+v) D_{1}}{805 a^{2}}\right) \cdot W^{2}-\left(\frac{(1+v) D_{1}}{3 R}\right) \cdot W \\
& +\left(\frac{29(1+v) D_{1}}{1680 \cdot a^{2}}\right) \cdot C^{2}-\frac{F_{m}}{1-v}
\end{aligned}
$$

where $F_{m}$ represents the term related to thermal effects, obtained by aid of Eqs. (11). Therefore, there are two different conditions to investigate: freely movable and immovable boundary edges; the second condition is divided into two conditions: existence of external pressure with and without temperature. Thermal effects can be considered in two different conditions: uniform temperature rise and temperature gradient along the thickness. In the first condition, the temperature changes between top and bottom surfaces $\Delta T=T_{c}-T_{m}$ and is uniform across the thickness. In the second condition, temperature varies with the rate of power-law distribution along the thickness. Substituting $\Delta T$ resulted from each of these conditions into Eqs. (11), thermal parameter, $F_{m}$, will be obtained. 


\subsection{Uniform temperature rise}

In this case, change of temperature is assumed to be uniformly distributed along the thickness. Substituting constant $\Delta T$ into Eqs. (11) results in $F_{m}$ as follows:

$$
F_{m}=P h \Delta T
$$

where [10]:

$$
P=E_{m} \alpha_{m}+\frac{E_{m} \alpha_{m}+E_{c m} \alpha_{c m}}{k+1}+\frac{E_{c m} \alpha_{c m}}{2 k+1} .
$$

This calculation is similar to the calculation of reference [10]. The present study is based on first-order shear deformation theory for thin and moderately thick shells, and the base of study in reference [10] is a classical theory for thin spherical shells; however, the calculation of the thermal parameter $F_{m}$, presented in Eqs. (11), is based on CLT theory; therefore, the results could be verified with those given in [10].

\subsection{Temperature gradient along the thickness}

In this case, change of temperature is assumed to be variable along the thickness according to the onedimensional Fourier equation of steady-state heatconduction established in a spherical coordinate system, as described in $[10,11]$. The origin of this coordinate system is the centre of a complete sphere as:

$$
\begin{aligned}
& \frac{d}{d \rho}\left[K(\rho) \frac{d T(\rho)}{d \rho}\right]+\frac{2 K(\rho)}{\rho} \frac{d T(\rho)}{d \rho}=0, \\
& T(\rho=R-h / 2)=T_{m}, \quad T(\rho=R+h / 2)=T_{c} .
\end{aligned}
$$

The thermal boundary conditions and frequent integration of the differential Fourier equation of steadystate heat-conduction in Eqs. (31) yield function of temperature change along the thickness based on radius direction:

$$
\Delta T(\rho)=\frac{\Delta T}{\int_{R-h / 2}^{R+h / 2} \frac{d \rho}{\rho^{2} K(\rho)}} \int_{R-h / 2}^{\rho} \frac{d \tau}{\tau^{2} K(\tau)} .
$$

As mentioned, the calculation of thermal parameter $F_{m}$ does not depend on the base theory (i.e., CLT or FTSD); therefore, in this case, it is possible to use the results from [10]. Because of the difficulties in the calculations in this case, only linear distribution of metal and ceramic constituents, i.e. $k=1$, is considered:

$$
K(\rho)=K_{m}+K_{c m} \cdot\left(\frac{2(\rho-R)+h}{2 h}\right),
$$

where $z$ is replaced by $\rho-R$. By plugging Eq. (33) into Eq. (32) and making integration, temperature distribution along the thickness direction will be given as [10]:

$$
\begin{aligned}
\Delta T(z)= & \frac{\Delta T}{I}\left[\frac{4 K_{c m}}{\left(K_{c}+K_{m}+2 K_{c m} S h\right)^{2}}\right. \\
& {\left[\ln \frac{\left(K_{c}+K_{m}\right) h+2 K_{c m} z}{2 h K_{m}}-\ln \frac{2(R+z)}{2 R-h}\right] } \\
& \left.+\frac{2(2 z+h)}{\left(K_{c}+K_{m}-2 K_{c m} S h\right)(R+z)(2 R-h)}\right]
\end{aligned}
$$

where $S h=R / h$ and $\rho$ is replaced by $z+R$ and [10]:

$$
I=\frac{4 K_{c m}}{\left(K_{c}+K_{m}-2 K_{c m} S h\right)^{2}} \ln \frac{K_{c}(2 S h-1)}{K_{m}(2 S h+1)} .
$$

Introduction of Eq. (34) into Eq. (11) gives the thermal parameter $F_{m}$ as [10]:

$$
F_{m}=\frac{\Delta T h L}{I},
$$

where [10]:

$$
\begin{aligned}
L= & \frac{K_{c m} \beta}{J^{2}}\left[\xi\left(S h+\frac{1}{2}\right)-1\right]-\frac{\eta}{2 J}\left(\xi-\frac{2}{2 S h-1}\right) \\
& +\frac{\eta}{J^{2}}\left(K_{m}-K_{c}+K_{c} \ln \frac{K_{c}}{K_{m}}\right) \\
& -\frac{K_{c m} \delta}{J^{2}}\left[S h-\left(S h^{2}-\frac{1}{4}\right) \xi\right]-\frac{\delta}{J}(1-S h \xi) \\
& -\frac{\delta}{2 J^{2} K_{c m}}\left[K_{m}^{2}-K_{c}^{2}+2 K_{m} K_{c} \ln \frac{K_{c}}{K_{m}}\right] \\
& +\frac{K_{c m} E_{c m} \alpha_{c m}}{J^{2}}\left[\frac{1}{9}+\frac{4 S h^{2}}{3}-\xi\left(\frac{1}{6}+\frac{4 S h^{3}}{3}\right)\right] \\
& +\frac{2 E_{c m} \alpha_{c m}}{J}\left[\frac{1}{6(2 S h-1)}+S h-S j^{2} \xi\right] \\
& +\frac{E_{c m} \alpha_{c m}}{9 J^{2} K_{c m}^{2}}\left[4\left(K_{m}^{3}-K_{c}^{3}\right)\right. \\
& \left.+3 K_{c}\left(K_{c}^{2}+3 K_{m}^{2}\right) \ln \frac{K_{c}}{K_{m}}\right],
\end{aligned}
$$

and:

$$
\begin{aligned}
J= & K_{c}+K_{m}-2 K_{c m} S h \quad \xi=\ln \frac{2 S h+1}{2 S h-1} \\
\eta= & \left(E_{c}+E_{m}\right)\left(\alpha_{c}+\alpha_{m}\right) \quad \delta=E_{c m}\left(\alpha_{c}+\alpha_{m}\right) \\
& +\alpha_{c m}\left(E_{c}+E_{m}\right) .
\end{aligned}
$$


By the aid of Eqs. (36) to (38), thermal parameter, $F_{m}$, is obtained considering the one-dimensional and the linear thermal gradient across the thickness.

$N_{r 0}$ is obtained by introducing the thermal parameter, $F_{m}$, obtained from Eqs. (29) or (36). Introducing $N_{r 0}$ into Eq. (25) completes the equation of equilibrium path which contains buckling and post-buckling of functionally-graded shallow thin and moderately-thick spherical shells subjected to uniform external pressure considering thermal effects incorporating two described boundary conditions.

\section{Validation of closed form results}

In this section, validation of results using the analytical formulation for mechanical and thermo-mechanical buckling and post-buckling of functionally-graded shallow spherical shells based on first-order transverse shear deformation theory is investigated by numerical simulation with the aid of finite elements software ABAQUS-6.10.1. The kind of elements used for modelling of the FGM shallow spherical shell is S4R elements, i.e. elements with 4 nodes and reduced integration considering transverse shear deformation based on first-order transverse shear deformation theory [17]. In this section, the accuracy of nonlinear finite-element method was carried out by its comparison with the experimental results, as indicated in [1] for isotropic shallow shells. Material and geometrical properties of the experimental specimens from [1] used in this section are demonstrated in Table 1.

In the present validation, Figures 3 and 4 demonstrate the accuracy of employed finite-element method in comparison with experimental results. Note that based on the assumption, the analytical manipulation in the current work is limited to the linear elastic behaviour. However, the experimental work in [1] requires incorporating both elastic and plastic phases of material properties. In the numerical analysis, the obtained result can be checked by experiments. The term $q_{c r}$ means classical buckling pressure $\left(q_{c r}=\right.$ $\left.2 E\left\{3\left(1-v^{2}\right)\right\}^{-1 / 2}[t / R]^{2}\right)$. Negligible difference be-

Table 1. Geometrical and mechanical properties of experimental models.

\begin{tabular}{cccccc}
\hline & \multicolumn{5}{c}{ Properties } \\
\cline { 2 - 6 } Model & $\boldsymbol{a}$ & $\boldsymbol{R}$ & $\boldsymbol{h}$ & $\boldsymbol{T}$ & $\boldsymbol{E}$ \\
& $(\mathbf{m m})$ & $(\mathbf{m m})$ & $(\mathbf{m m})$ & $\left({ }^{\circ} \mathbf{c}\right)$ & $\left(\mathrm{g} / \mathbf{c m}^{2}\right)$ \\
\hline B96 & 170 & 1853 & 1.98 & 20 & $3.1 \mathrm{E}+7$ \\
E90 & 170 & 1908 & 2.97 & 25 & $3.1 \mathrm{E}+7$ \\
\hline
\end{tabular}

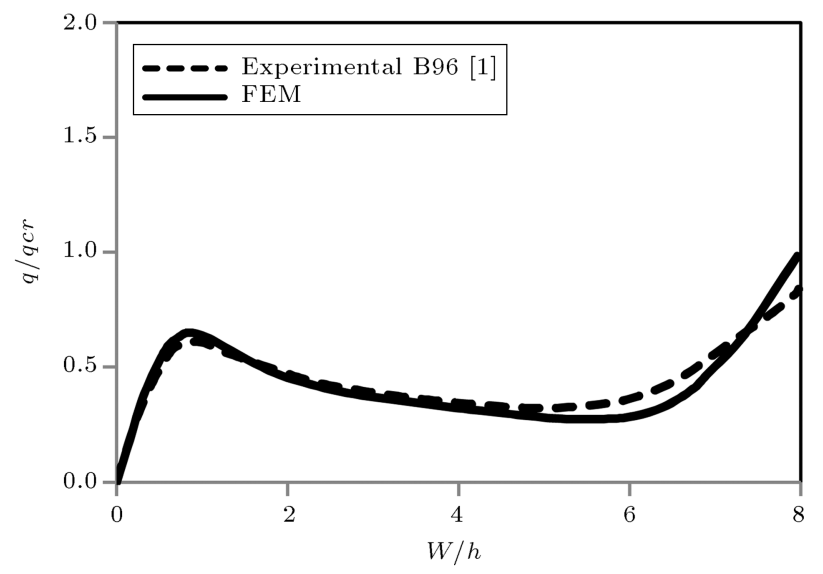

Figure 3. Comparing equilibrium path using the finite-elements method with experimental method in B-96 model [1].

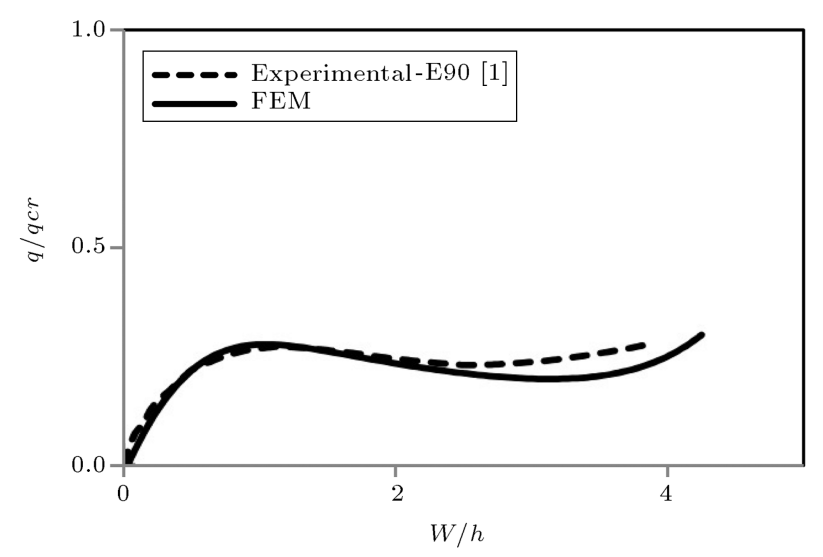

Figure 4. Comparing equilibrium path using the finite-element method with experimental method in E-90 model [1].

tween the equilibrium paths obtained by employing the finite-element method and existing experiments indicates sufficient accuracy of numerical simulation in the current study.

To illustrate the validity of the closed-form results obtained in the last part, it is proposed to consider the ceramic-metal functionally-graded shallow spherical shell with the properties mentioned in Table 2 [10].

The Poisson ratio is chosen to be constant and equal to 0.3. Two different conditions, i.e. thin and moderately thick FGM shells are considered in this section.

Figures 5 and 6 indicate the validation of the closed-form equilibrium path formulation using Eq. (25) for thin and moderately thick spherical with the numerical FEM. It is seen that the FSDT brings the

Table 2. FGM Material properties used for numerical studies and discussion.

\begin{tabular}{cccccc}
\hline $\boldsymbol{E}_{\boldsymbol{m}}$ & $\boldsymbol{E}_{\boldsymbol{c}}$ & $\boldsymbol{\alpha}_{\boldsymbol{m}}$ & $\boldsymbol{\alpha}_{\boldsymbol{c}}$ & $\boldsymbol{K}_{\boldsymbol{m}}$ & $\boldsymbol{K}_{\boldsymbol{c}}$ \\
\hline $70(\mathrm{Gpa})$ & $380(\mathrm{GPa})$ & $23 \times 10^{-6}\left({ }^{\circ} \mathrm{C}^{-1}\right)$ & $7.4 \times 10^{-6}\left({ }^{\circ} \mathrm{C}^{-1}\right)$ & $204(\mathrm{~W} / \mathrm{mK})$ & $10.4(\mathrm{~W} / \mathrm{mK})$ \\
\hline
\end{tabular}




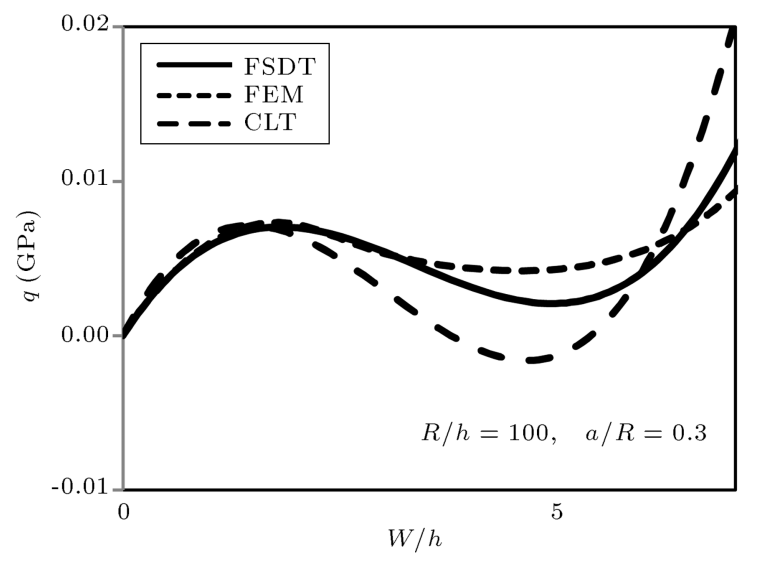

Figure 5. Comparison of results using the first-order shear deformation theory (FSDT), classical theory (CLT) [10], and finite-elements method for thin shell.

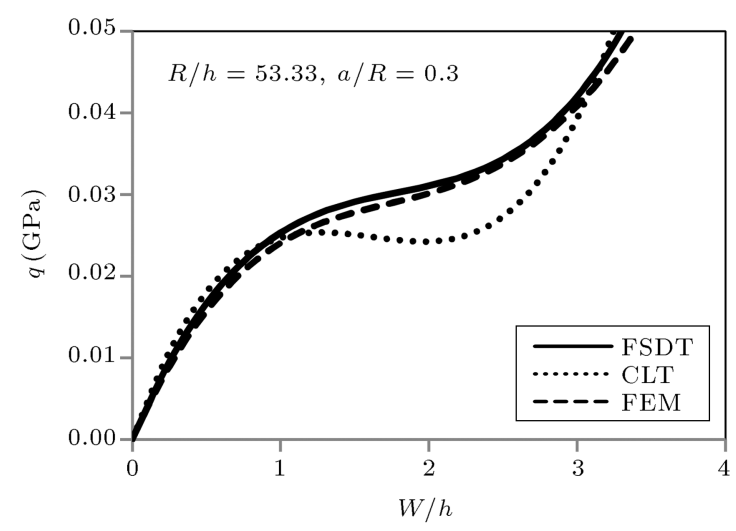

Figure 6. Comparison of results using the first-order shear deformation theory (FSDT), classical theory (CLT) [10], and finite-elements method for thick shell.

results closer to the numerical estimation as validated by the existing experiments in Figures 3 and 4 .

\section{Numerical results and discussion}

To illustrate the proposed method, consider a metalceramic FGM shallow spherical shell. The geometry and loading of the shell are demonstrated in Figure 1. The combination of materials consisting of steel and ceramic with temperature-independent Young's modulus, the thermal expansion coefficients, and thermal conductivity are supposed with those information as given in Table 2; Poisson's ratio is assumed to be 0.3 for the steel and ceramic. Clamped movable and clamped immovable supported boundary conditions are assumed. All of the results are presented in the form of load-deflection curves that show the equilibrium path.

Figures 7 and 8 show the load-deflection curves for thick and moderately thick shells $(R / h=53.33$ and 70). The effect of the transverse shear deformation on equilibrium path is evident in both cases as it is more pronounced in the thick shell as was expected.

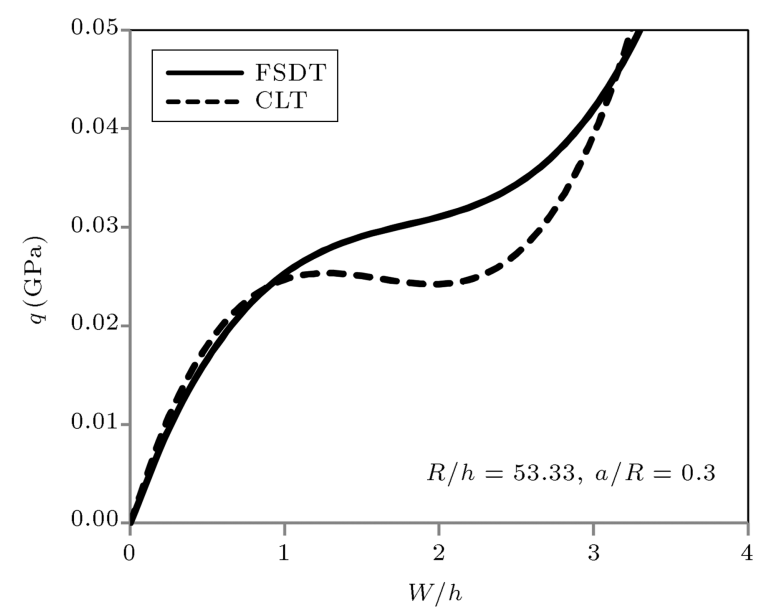

Figure 7. Comparison of results using the first-order shear deformation theory (FSDT), and classical theory (CLT) [10] for the thick shell.

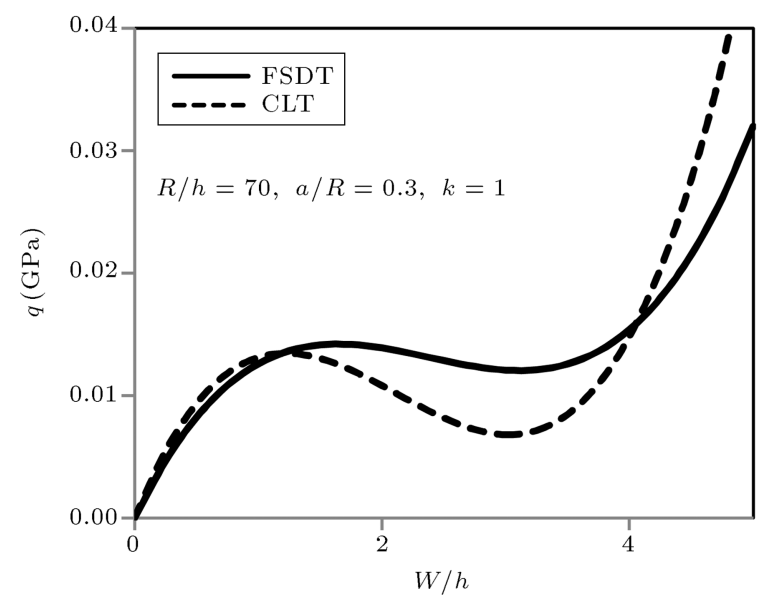

Figure 8. Comparison of results using the first-order shear deformation theory (FSDT), and classical theory (CLT) [10] for the moderately thick shell.

In these cases, the range of unstable zone obtained by the first-order theory is longer than classical ones, but the intensity of instability, i.e. difference between the maximum and minimum of load-deflection curves, is lower than the classical one due to the more flexibility in the FSDT case.

The above-mentioned difference is less distinct in the thin spherical shells as it is observable in Figure 9 for $(R / h=140)$.

Figure 10 indicates the effects of $a / R$ ratio on equilibrium path. Increasing the $a / R$ exhibits growing the difference between upper and lower limit buckling loads and range of the unstable zone.

Figure 11 illustrates the effects of transverse shear deformation on equilibrium paths in different cases of relative depth ratio $(a / R)$ and constant mechanical properties and relative thickness $(R / h)$ by means of FSDT in comparison to CLT. In all of the cases, it is clearly seen that by increasing the ratio of the nonlinear 


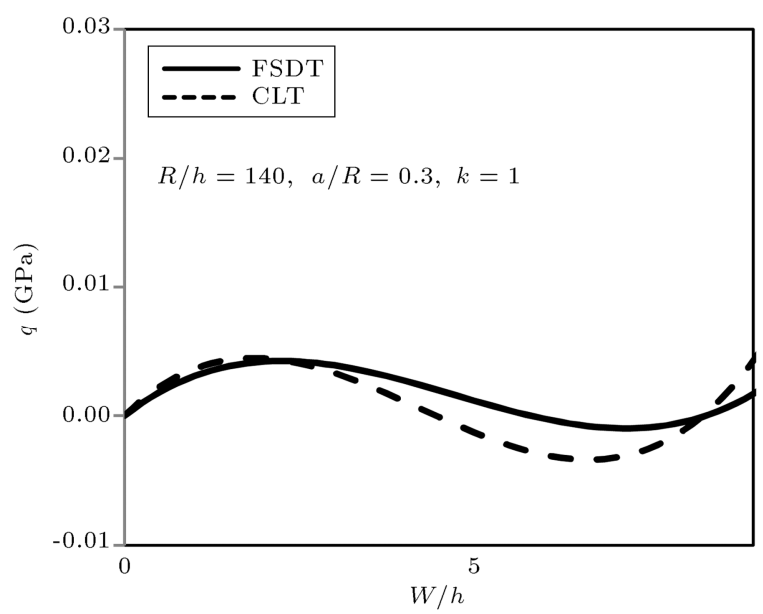

Figure 9. Comparison of results using the first-order shear deformation theory (FSDT), and classical theory (CLT) [10] for the thin shell.

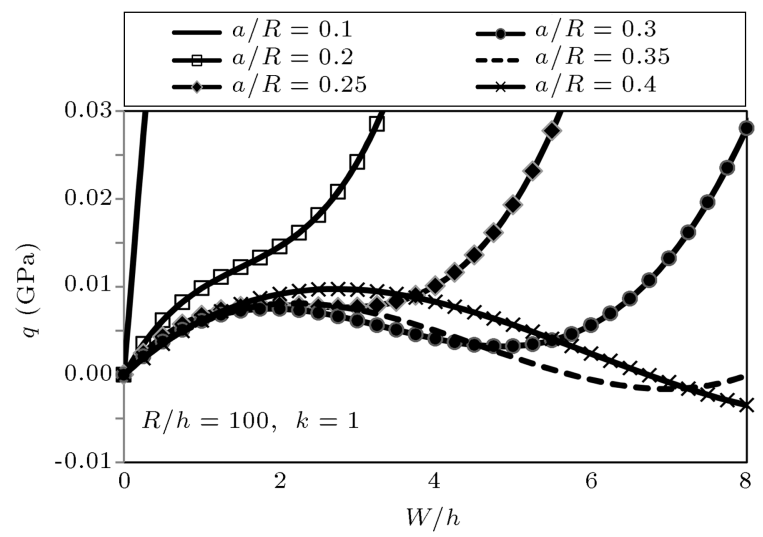

Figure 10. Effects of relative depth $(a / R)$ on equilibrium path.

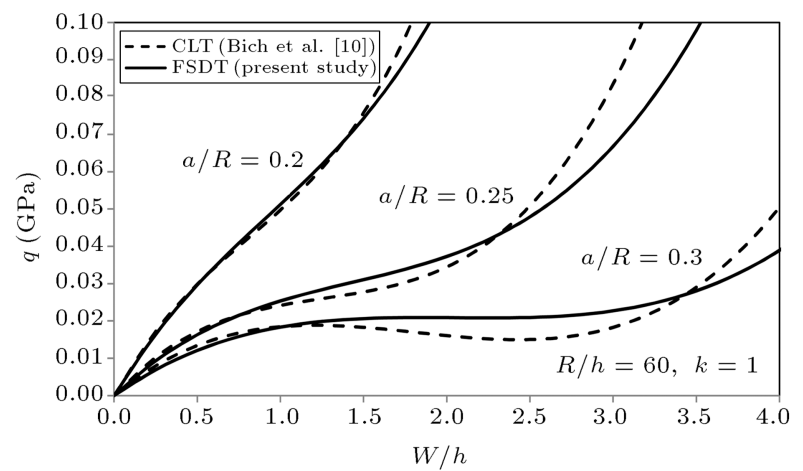

Figure 11. Comparison of the first-order transverse shear deformation theory (FSDT) and classical theory (CLT) in different relative depth $(a / R)$ for moderately thick shells.

behavior, results obtained by present study would be more evident. These differences are remarkable when the deflection of the shell is in the unstable zone, which is addressing the geometrical nonlinearity.

Figure 12 displays the effects of relative thickness on equilibrium paths in different cases of relative

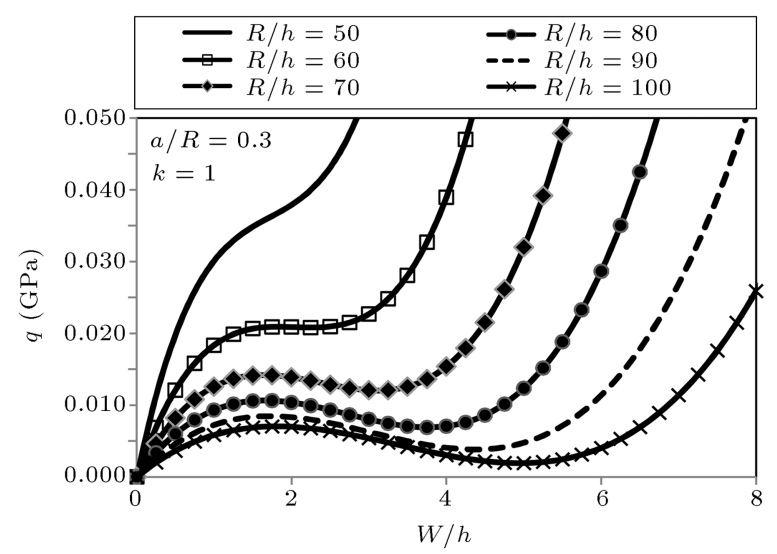

Figure 12. Effects of relative thickness on equilibrium path.

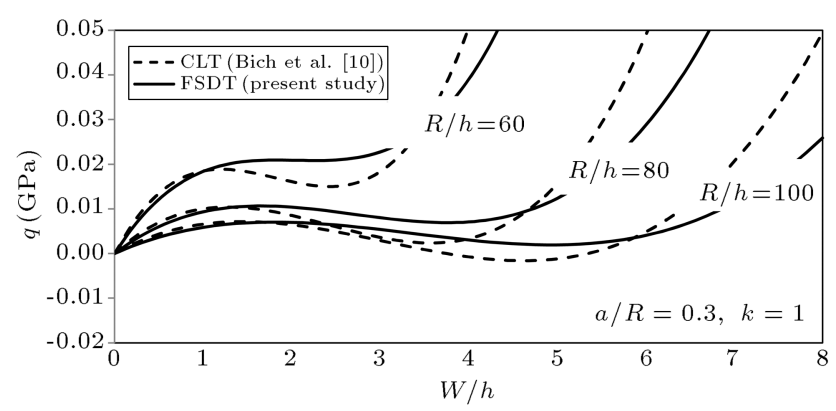

Figure 13. Comparison of the first-order transverse shear deformation theory (FSDT) and classical theory (CLT) in different relative thickness $(R / h)$.

thickness ratio $(R / h)$, while the mechanical properties and relative depth $(a / R)$ are considered to be constant in FSDT approach. In all of the cases, it is clear that by increasing the relative thickness $(R / h)$, the difference between upper and lower limit buckling loads would grow up as the intensity of snap-through behaviour becomes dominant. These differences are remarkable when the relative thickness is increasing.

Figure 13 compares the FSDT and CLT approaches for estimation of load deflection equilibrium path for different $(R / h)$ ratios. It is seen that as $R / h$ increases, the differences between the results obtained from two theories approach each other. It is observed that in all of the cases, that is, thin and moderately thick FGM shells, the main difference between results of the CLT and FSDT is in the unstable zone, such that the geometrical nonlinear behaviour is noticeable even in thin shells.

Figure 14 denotes the effects of transverse shear deformation on equilibrium paths in different arbitrary values for volume fraction indices $(k)$ while keeping constant the other mechanical and geometrical properties. As $k$ values increase, the difference between equilibrium paths obtained by CLT and FSDT approaches each other.

By considering the temperature-independent me- 


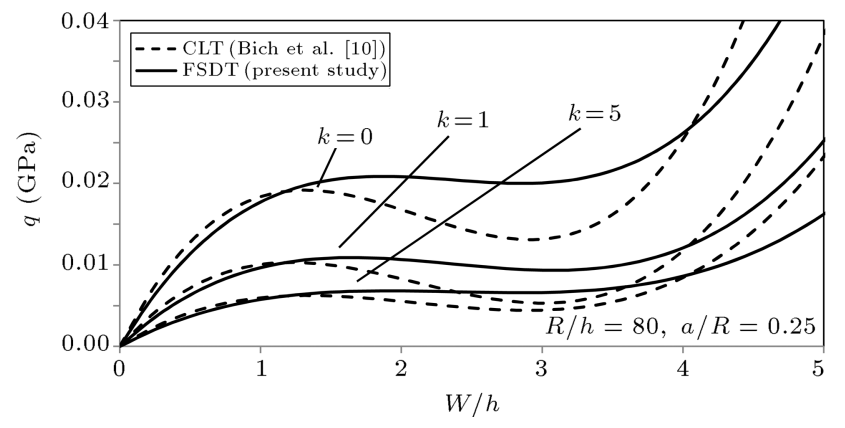

Figure 14. Comparison of the first-order transverse shear deformation theory (FSDT) and classical theory (CLT) in different $r$ volume fraction index $(k)$.

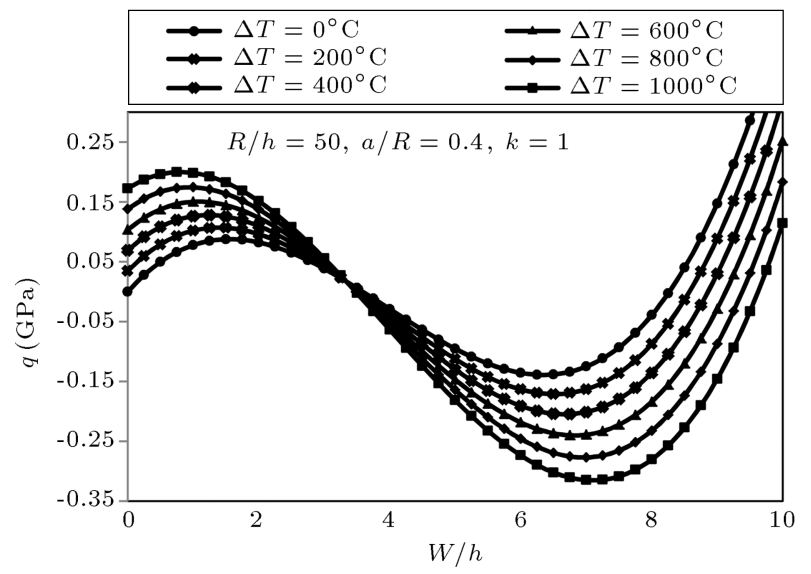

Figure 15. Effects of temperature on equilibrium path in uniform distribution of temperature along the thickness.

chanical properties, in the case of fixed clamped edges, rising the environment temperature prior to applying external pressure results outward deflection (negative deflection). This negative deflection results in bifurcation-type buckling behaviour due to the presence of temperature field. This bifurcation point is located at intersection of equilibrium path's curve with $q$ axis, where the negative deflection finishes and the positive deflection begins. Figure 15 displays this effect in case of uniform distribution of temperature across the thickness, whereas Figure 16 displays this effect in the case of existence of temperature gradient along the thickness. All of the curves in Figures 15 and 16 are plotted for 6 different temperature changes. As it is shown in these figures, rising the environment temperature results in increasing the upper limit and decreasing the lower limit. It is interesting to note that the severity of this effect in the case of existence of temperature gradient along the thickness is lower than the case of uniform temperature distribution along the thickness because of the low value for the ceramic thermal conductivity $\left(K_{c}\right)$.

Equilibrium paths in the case of uniform distribution of temperature along the thickness in comparison with the case of existence of temperature gradient

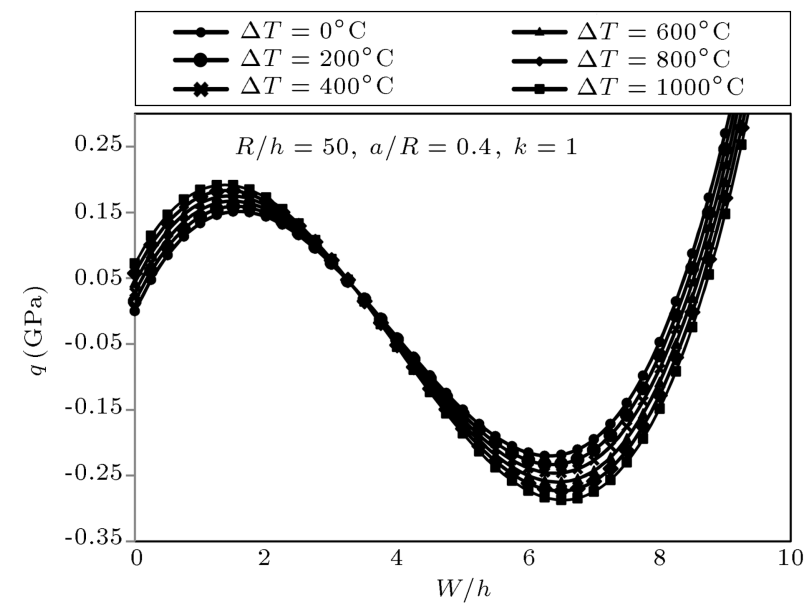

Figure 16. Effects of temperature on equilibrium path through the thickness temperature gradient.

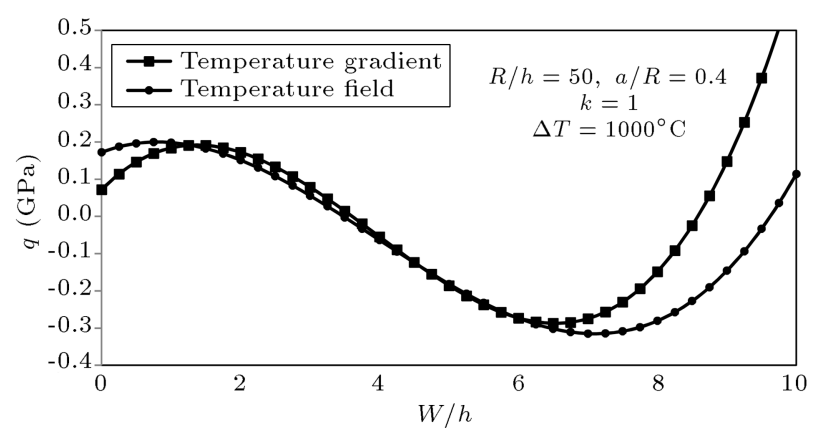

Figure 17. Equilibrium path in the case of uniform distribution of temperature along the thickness in comparison with the case of existence of temperature gradient.

are demonstrated in Figure 17. It is evident that the intensity of snap-through behaviour, upper limit buckling load, and the deflection of the unstable zone in the uniform temperature distribution is notably more than the case of temperature gradient.

Figures 18 and 19 compares the FSDT and CLT estimations of the upper and lower limit loads in the load deflection equilibrium path when the thermal effects are considered for thin and moderately thick FGM shells. The curves are plotted for $\Delta T=200^{\circ} \mathrm{C}$. It is important to note that differences between the FSDT and CLT for the thick shell theories are more important than for the thin shell theories, especially in the unstable zone that the equilibrium path has been experiencing geometrical nonlinearity behaviour.

\section{Conclusion remarks}

In the current paper, the axsisymmetrical nonlinear response of a clamped functionally-graded shallow spherical shell subjected to uniform external pressure, considering the effects of thermal stresses, is investigated using First-Order Shear Deformation Theory (FSDT). 


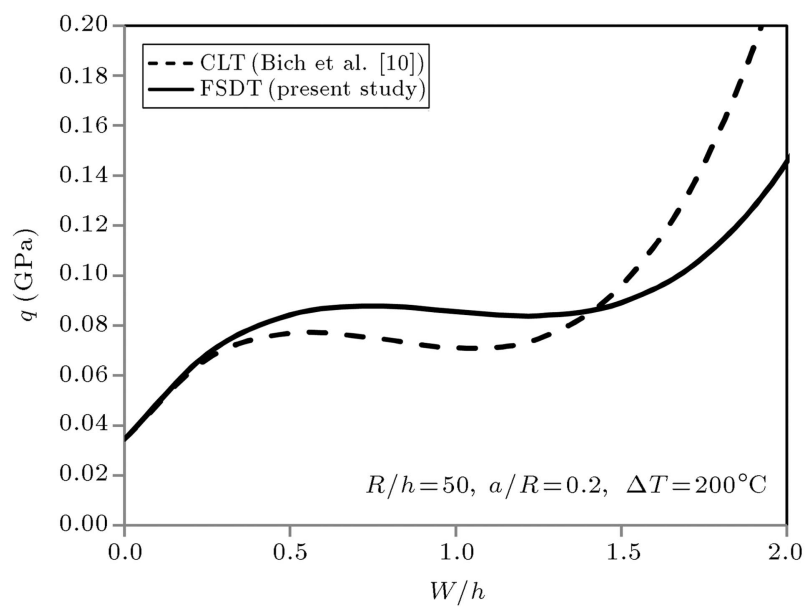

Figure 18. Effects of transverse shear deformation on equilibrium path obtained by first-order transverse shear deformation theory (FSDT) in comparison with classical theory (CLT) considering thermal effects (uniform temperature distribution) for thick FGM shells.

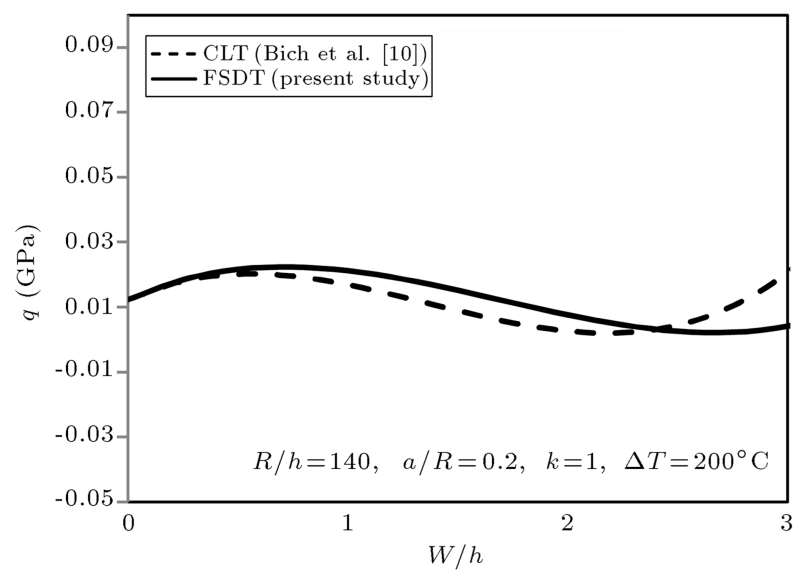

Figure 19. Effects of transverse shear deformation on equilibrium path obtained by first-order transverse shear deformation theory (FSDT) in comparison with classical theory (CLT) considering thermal effects (uniform temperature distribution) for thin FGM shells.

Effects of considering the degree of freedom related to the transverse shear deformation on the equilibrium path incorporating the buckling and post-buckling of the mentioned shell is the main novelty of the present study. To investigate the effects of shear deformation on equilibrium path, all of the cases obtained by firstorder shear deformation theory are compared with the classical results obtained in [10]. On the other hand, by mentioning the assumptions, the equilibrium path is presented in the closed-form formula depending on mechanical and geometrical properties and boundary condition of the shell. The analytical solution was verified by the numerical simulation using the FEM approach in the ABAQUS6.10.1. The numerical results were also validated by existing experiments as found in [1]. Numerical results showed that diffrerences be- tween CLT and FSDT are more visible in unstable zone by changing the mechanical and geometrical properties. Decreasing the relative thickness $(R / h)$ and volume fraction index $(k)$ results in more different equilibrium paths. This phenomenon is so because of the effect of transverse shear deformation that is more effective in cases that the stiffness of the shell is in higher levels. For this condition, the use of the CLT results in more intensive unstability, because one of the rotational degrees of freedom of the shell is restricted and the unstabale deformations are more intensive. But, by using the FSDT, this restricted rotational degree of freedom is controlled by considering the transverse shear strain, and the intensity of unstability will be decreased.

Increasing the relative depth ratio $(a / R)$ and temprature change $(\Delta T)$ resultsin different equilibrium paths for CLT and FSDT. These differences can be vindicated using the last explanation.

\section{References}

1. Yamada, Seishi and Uchiyama, Kazuo and Yamada, Motohiko "Experimental investigation of the buckling of shallow spherical shells", Nonlinear Mechanics, 18, pp. 37-54 (1983).

2. Li, Q.S., Liu, J. and Tang, J. "Buckling of shallow spherical shells including the effects of transverse shear deformation", Mechanical Sciences, 45, pp. 1519-1529 (2003).

3. Lei, X.Y., Huang, M.K. and Wang, X. "Analysis of the explicit fundamental solution of a shallow spherical shell involving shear deformation", Applied Mathematical Modeling, 19, pp. 194-200 (1995).

4. Cohen, G.A. "Buckling of laminated anisotropic shells including transverse shear deformation", Computer Methods in Applied Mechanics and Engineering, 26, pp. 197-204 (1981).

5. Carrera, E. "The effect of shear deformation and curvature on buckling and vibration of cross-ply laminated composite shells", Sound and Vibration, 150(3), pp. 405-433 (1991).

6. Nie, G.H. "Asymptotic buckling analysis of imperfect shallow spherical shells on non-linear elastic foundation", Mechanical Sciences, 43, pp. 543-555 (2001).

7. Reddy, J.N. and Liu, C.F. "A higher-order shear deformation theory of laminated elastic shells", Engineering Sciences, 23, pp. 319-330 (1981).

8. Ferreira, A.J.M., Roque, C.M.C. and Jorge, R.M.N. "Modelling cross-ply laminated elastic shells by a higher-order theory and multi-quadrics", Computers and Structures, 84, pp. 1288-1299 (2006).

9. Hafealkotob, A. and Eslami, M.R. "Thermomechanical buckling of simply supported shallow FGM spherical shells with temperature-dependent material", Iranian Journal of Mechanical Engineering, 11, pp. 41-65 (2010). 
10. Bich, D.H. and Tung, H.V. "Non-linear axisymmetric response of functionally graded shallow spherical shells under uniform external pressure including temperature effects", International Journal of Non-Linear Mechanics, 46, pp. 1195-1204 (2011).

11. Mao, Y.Q., Fu, Y.M., Chen, C.P. and Li, Y.L. "Nonlinear dynamic response for functionally graded shallow spherical shell under low velocity impact in thermal environment", Applied Mathematical Modelling, 35, pp. 2887-2900 (2011).

12. Zenkour, A.M. and Sobhy, M. "Thermal buckling of various types of FGM sandwich plates", Composite Structures, 93, pp. 93-102 (2010).

13. Shariyat, M. "Dynamic buckling of suddenly loaded imperfect hybrid FGM cylindrical shells with temperature-dependent material properties under thermo-electro-mechanical loads", International Journal of Mechanical Sciences, 50, pp. 1561-1571 (2008).

14. Shariyat, M. "Vibration and dynamic buckling control of imperfect hybrid FGM plates with temperaturedependent material properties subjected to thermoelectro-mechanical loading conditions", Composite Structures, 88, pp. 240-252 (2009).

15. Chung, Y.L. and Chen, W.T. "Bending behavior of FGM-coated and FGM-undercoated plate with two simply supported opposite edges and two free edges", Composite Structures, 81, pp. 157-167 (2007).
16. Zhao, X., Lee, Y.Y. and Liew, K.M. "Mechanical and thermal buckling analysis of functionally graded plates", Composite Structures, 90, pp. 161-171 (2009).

17. ABAQUS User's Manual, ABAQUS, Inc. (2003).

\section{Biographies}

Mohammad Amin Shahmohammadi received his BS degree from Azad Islamic University-South of Tehran branch, Tehran, Iran, and his MS degree from Amirkabir University of Technology, Tehran, Iran. He is currently a $\mathrm{PhD}$ degree candidate in the Department of Civil Engineering in Isfahan University of Technology, Isfahan, Iran. His research interests include thin-walled structures, composite structures, structural analysis using FEM, analysis of structures made of Functionally Graded Materials (FGMs), especially in shell and plates.

Mohammad Zaman Kabir is a Professor in Solid Mechanics and Composite Materials in the Department of Civil and Environmental Engineering, Amirkabir University of Technology, Tehran, Iran. His research interests are structural stability, structural analysis using FEM, experimental methods in structural engineering, composite materials, structural optimization, damage detection, and rehabilitation of structures. 